\title{
A 2D Electromagnetic PIC Code for Distributed Memory Parallel Computers
}

\author{
T. Krücken \\ California Institute of Technology, Pasadena, CA 91125 \\ P. C. Liewer and R. D. Ferraro \\ Jet Propulsion Laboratory, California Institute of Technology, Pasadena, CA 91109 \\ V. K. Decyk \\ Physics Department, University of California, Los Angeles, CA 90024
}

\begin{abstract}
The two dimensional electrostatic plasma particle in cell (PIC) code described in [1] has been upgraded to a $2 D$ electromagnetic PIC code running on the Cabtech/JPL Mark IIIfp and the Intel iPSC/860 parallel MIMD computers. The code solves the complete time dependent Maxwell's equations where the plasma responses, i.e., the charge and current density in the plasma, are evaluated by advancing in time the trajectories of $\sim 10^{6}$ particles in their self-consistent electromagnetic field. The field equations are solved in Fourier space. Parallelisation is achieved through domain decomposition in real and Fourier space. Results from a simulation showing a two-dimensional Alfvèn wave filamentation instability are shown; these are the first simulations of this $2 D$ Alfvèn wave decay process.
\end{abstract}

\section{Introduction}

In electromagnetic particle-in-cell (PIC) simulations, a plasma is modeled by advancing $10^{3}-10^{6}$ particles (electrons and ions) in their self-consistent electromagnetic field plus a uniform static external magnetic field [2]. The fields are found by solving Maxwell's equations on a discrete grid with the plasma current and charge densities as sources. At each timestep Newton's equations of motion are solved for all particles to update their positions and velocities, which are then used to evaluate the charge and current densities at the grid points needed to solve Maxwell's equations for the electromagnetic fields. The field equations are integrated using a Fourier decomposition in the two spatial dimension assuming periodic boundary conditions and a leap-frog method in time. To obtain efficient operation on a distributed memory parallel computer the physical domain is divided in one dimension in sub-domains equal in number to the number of processors so that each sub-domain contains the same number of grid points and roughly the same number of particles [3]. Since in the present Alfvèn wave application no major density inhomogeneities occurred, it was not necessary to move the sub-domain boundaries during the runs in order to maintain load balance among the processors (compare ref. [4] for dynamic load balancing in PIC codes). The code runs on the Caltech/JPL Mark IIIfp and the Intel iPSC/860 Hypercube distributed memory computers.

The code can be used to study wave-particle interactions and instabilities in the linear and nonlinear phase in plasmas. In the first applications we simulated the decay of large amplitude Alfvèn waves and compared our results with the decay instabilities predicted by theoretical models based on linearized two fluid MHD models [5,6]. Large amplitude Alfvèn waves are observed in the solar wind, upstream of the Jovian bow shock and near other interplanetary shocks. Understanding the decay of these waves will help understand spacecraft observations and increase our understanding of low frequency plasma magnetic turbulence.

In Section 2 we will describe the numerical method; Section 3 contains a discussion of the parallel aspects of the algorithm including some performance figures. In Section 4 we will briefly outline the various theories of parametric instabilities of high amplitude Alfvèn waves in the solar wind before showing results of our simulations. 


\section{An Electromagnetic Particle-in-Cell Code}

The code solves the equation of motion for the plasma particles and the full Maxwell's equations for the electromagnetic field. [2]. A particle with velocity $\vec{v}_{p}$ at position $\vec{x}_{p}$ is subject to the Lorentz force:

$$
\begin{gathered}
\frac{d \vec{v}_{p}}{d t}=\frac{q_{p}}{m_{p}}\left(\vec{E}\left(\vec{x}_{p}\right)+\frac{\vec{v}_{p}}{c} \times\left(\vec{B}\left(\vec{x}_{p}\right)+\vec{B}_{0}\right)\right), \\
\frac{d \vec{x}_{p}}{d t}=\vec{v}_{p} .
\end{gathered}
$$

$\vec{B}_{0}$ is a constant uniform external magnetic field, $\vec{E}$ and $\vec{B}$ describe the self-consistent electromagnetic field which is a solution of Maxwell's equations. Variations are allowed in two directions $\left(\vec{e}_{x}\right.$ and $\left.\vec{e}_{y}\right)$, but all three componets of $\vec{v}, \vec{E}$, and $\vec{B}$ are needed. A spectral method is used to solve the field equations on a discrete grid with periodic boundary conditions (because of the explicit time dependence of the equations it is very difficult to implement other boundary conditions ). In Fourier space Maxwell's equations can be split in a transverse and a longitudinal part:

$$
\begin{gathered}
\frac{1}{c} \frac{d \vec{B}(\vec{k}, t)}{d t}=-i \vec{k} \times \vec{E}_{t r}(\vec{k}, t) \\
\frac{d \vec{E}_{t r}(\vec{k}, t)}{d t}=i c \vec{k} \times \vec{B}(\vec{k}, t)+4 \pi \vec{J}_{t r}(\vec{k}, t) \\
\vec{E}_{l o n}(\vec{k}, t)=-4 \pi \frac{i \vec{k}}{k^{2}} \rho(\vec{k}, t)
\end{gathered}
$$

where

$$
\begin{gathered}
\vec{A}(\vec{x})=\sum_{k_{x}, k_{y}} \vec{A}\left(k_{x}, k_{y}\right) \cdot e^{i\left(k_{x} x+k_{y} y\right)} \\
\vec{k}=k_{x} \vec{e}_{x}+k_{y} \vec{e}_{y} .
\end{gathered}
$$

The transverse and longitudinal components of a mode of $\vec{A}$ are defined as

$$
\begin{gathered}
\vec{A}_{l o n}(\vec{k}, t)=\frac{\vec{k}(\vec{k} \cdot \vec{A}(\vec{k}, t))}{k^{2}}, \\
\vec{A}_{t r}(\vec{k}, t)=\vec{A}(\vec{k}, t)-\vec{A}_{l o n}(\vec{k}, t)
\end{gathered}
$$

so that $\vec{k} \cdot \vec{A}_{t r}(\vec{k}, t)=\vec{k} \times \vec{A}_{\text {lon }}(\vec{k}, t)=0$. The source terms in Maxwell's equations, $\rho=\frac{1}{V} \sum_{p} q_{p}$ and $\vec{J}=\frac{1}{V} \sum_{p} q_{p} \vec{v}_{p}$, are the charge and current density respectively due to the plasma particles, with $V$ the simulation volumn.
Each time step in the PIC code proceeds in two stages. In the first stage, the particle equations are solved. The fields at the position of the particle is found by interpolation from the fields at the grid points. The new charge and current densities are found by interpolation of the particle information to the grid points. A quadratic interpolation scheme is used. In the second stage, the charge and current densities are Fourier-transformed and the field equations solved using an explicit leapfrog algorithm. The new fields are transformed back to real space in preparation for the next set of particle updates.

Runtime diagnostics include evaluation of particle distribution functions, particle, energy and momentum flux, drift velocities, overall momentum and energy, various plots of the electromagnetic fields, as well as evaluation of the field momentum and energy. These diagnostic, including the plots, are all performed in parallel.

\section{Parallel aspects of the Code}

The code runs SPMD (single program multiple data -all node programs are identical) style under Fortran Cubix on the Mark IIIfp and under Express Fortran Cubix on the Intel. Parallelisation is achieved by means of one dimensional domain decomposition in real and Fourier space. Each processor knows the particles and grid quantities in his subdomain in real space $(x, y)$, which is a horizontal strip. In Fourier space $\left(k_{x}, k_{y}\right)$ the subdomains are vertical strips. These decompositions are described in Ref. 1.

Interprocessor communication is necessary at several stages. The 2D FFT is done as two sets of $1 \mathrm{~d}$ FFTs, first in the horizontal, then in the vertical direction. Between these two operations the entries $f\left(k_{x}, y\right)$ are rearranged among processors, such that each set of 1d FFTs can be done in parallel. This requires considerable interprocessor communication. Information about particles moving into a neighbouring processor's subdomain must also be passed to the new processor's particle list and removed from the old processor's list. Additional communication is also necessary for the diagnostics.

Most of the computational time is needed to advance the particles. Due to the nonuniformity of the magnetic field the particle push is in an electromagnetic code much more time consuming than in an electrostatic PIC code. To obtain efficient operation it is therefore very important that the number of particles in each processor is roughly the same. 
The code has been run on the Caltech/JPL Mark IIIfp (up to 128 processors) with the Weitek XL processor and the Intel iPSC/860 (up to 64 processors). A fixed problem on the same number of nodes runs roughly three times faster on the Intel iPSC/860 than on the Mark IIIfp: For a problem with 294,912 particles and a $256 \times 256$ grid run for 150 times steps on 32 processors, the Intel total time was 373 secs versus a Mark IIIfp time of 1043 secs.

\section{Application to 2D Decay of Large Amplitude Alfvèn Waves}

The code can be used to study wave phenomena, wave-particle interactions, kinetic effects, and instabilities in the linear and nonlinear regimes in rectangular 2D geometry. In the first applications we have used it to simulate the behaviour of large amplitude circularly polarised parallel (to the static magnetic field) propagating Alfvèn waves as observed in the solar wind and other interplanetary environments. These waves are subject to parametric decays into other plasma waves caused by nonlinear terms: The initial "pump" Alfvèn wave $\left(\omega_{0}, \vec{k}_{0}\right), \vec{k}_{0} \| \vec{B}_{0}$ beats with a daughter wave $(\omega, \vec{k})$ which is initially excited from the noise and forms a pair of sideband waves $\left(\omega_{ \pm}, \vec{k}_{ \pm}\right)$. Frequency and wavenumber matching requires that $\left(\omega_{ \pm} \approx \omega_{0} \pm \omega, \vec{k}_{ \pm} \approx \vec{k}_{0} \pm \vec{k}\right)$. If all waves are normal modes (eigenmodes), i.e., all pairs $\omega, \vec{k}$ are solutions of the plasma dispersion relation, the instability has a large growth rate. For high amplitude pump waves it is, however, oftcn sufficient that three waves are eigenmodes.

Parametric instabilities of Alfvèn waves have been discussed in numerous theoretical papers based on plasma fluid equations (sce for example $[5,6]$ ) and the papers cited there). The important processes for our simulations are the decay instability and the filamentation instability. In the first case, the daughter wave is an ion sound wave with a shorter wavelength than the pump Alfvèn wave and the sidebands are electromagnetic waves. The decay products can propagate parallel to the pump wave or at oblique angles; generally the fastest decay occurs for parallel decay products. The one dimensional decay processes can be studied in simpler 1D models.

On the other hand, the filamentation instability can not be treated in a one dimensional model since the daughter "wave" is a purely growing, magnetostatic perturbation, i.e. $\operatorname{Re}(\omega) \simeq 0$, with $\vec{k} \perp \vec{k}_{0}$. The pump wave gets "ducted" in density/B-field filaments due to this magnetostatic perturbation. Sidebands are obliquely propagating electromagnetic waves. This wave has only recently been discussed theoretically $[6]$. Most theoretical study of these decay processes are limited to linearized fluid treatments; particle simulations are used to include kinetic and nonlinear effects on the evolution of these processes. Here, results from the first $2 \mathrm{D}$ simulations showing the 2D filamentation instability are presented.

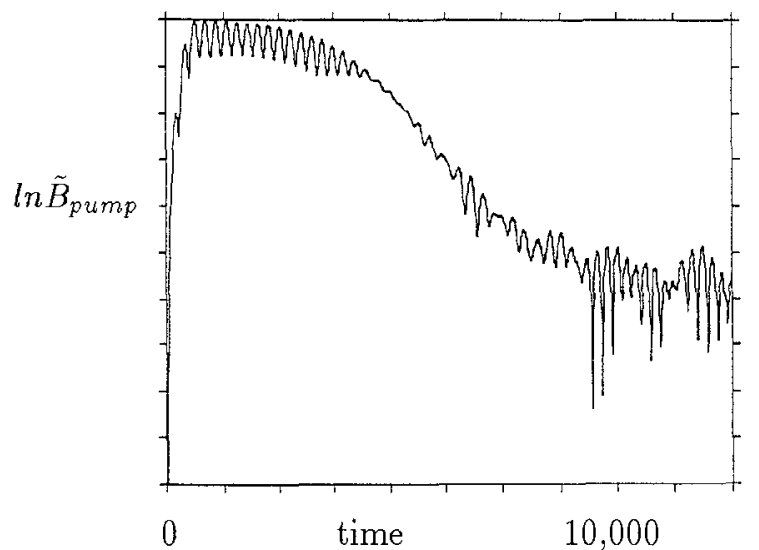

Fig. 1 Time history of the amplitude of the magnetic perturbation of the pump wave.

Figure 1 shows the time history of the amplitude of the pump wave in a simulation with a right hand circularly polarised Alfvén wave of amplitude $\tilde{B} / B^{0} \approx 0.5$ propagating parallel to an external magnetic field $B^{0} \vec{e}_{y}$. For this run, $\beta$ (ratio of plasma to magnetic pressure) $=0.3, m_{i} / m_{e}=16, k_{y}^{0} V_{A} / \omega_{c i} \approx 0.3$, where $V_{A}$ is the Alfvèn speed, and $\omega_{c e} / \omega_{p e}=1$. The initial pump Alfvèn wave is excited by applying an external oscillatory current for a short time at the beginning of the run (Fig. 1). This pump wave is then observed to decay in time, transferring its energy to other modes and to the plasma particles. In the computer simulations, many different decay processes occur simultaneously. The various decay instabilities are separated by using a post processor to analyze the time history and power spectrum of various Fourier modes.

At early times in this simulation, the dominant decay process was a 1D decay instability, with the daughter wave an ion acoustic wave with $k_{y}^{d}=1.5 k_{y}^{0}$ and an Alfvèn wave sideband with $k_{y}^{s}=0.5 k_{y}^{0}$; power spectra from the post processor show that these waves also satisfy the frequency matching condition $\omega^{0} \approx \omega^{d}+\omega^{s}$ with $\omega^{0} / \omega_{c i} \approx 0.45, \omega^{d} / \omega_{c i}=0.4$, and $\omega^{s} \approx 0.005$. The grow rate of this decay instability is $\gamma / \omega_{r} \simeq 0.1$, consistent with theoretical predictions for similar parameters[6].

In addition to the $1 \mathrm{D}$ decay processes, two $2 \mathrm{D}$ fil- 
amentation decays are observed to grow with lower growth rates than the dominant $1 \mathrm{D}$ decay process. Because of the several competing processes, the growth phase observed for these processes are not purely exponential. These processes are identified as filamentation instabilities because, at early times, a perpendicularly propagating daughter wave with zero frequency is observed to grow more or less simultaneously with an obliquely propagating wave with a frequency approximately equal to the pump wave. Both daughtel' and sideband waves have both density and magnetic perturbations as expected for these electromagnetic modes. For one filamentation instability, the zero frequency daughter wave has $\vec{k}_{\perp}=2 k^{0} \vec{e}_{x}$, the sideband has $\vec{k}=2 k^{0} \vec{e}_{x}+k^{0} \vec{e}_{y}$, and the growth rate $\gamma / \omega^{0} \sim 0.08$. For the other filamentation instability, the zero frequency daughter wave has $\vec{k}_{\perp}=k^{0} \vec{e}_{x}$, the sideband has $\vec{k}=k^{0} \vec{e}_{x}+k^{0} \vec{e}_{y}$, and the growth rate $\gamma / \omega^{0} \sim 0.05$. These growth rates are of comparable magnitude to theoretical predictions $[6]$. Other waves are also excited in the course of the simulation; even at relatively early times, the situation in these fully nonlinear simulations is far from the ideal situation assumed in the linear analysis $[5,6]$.

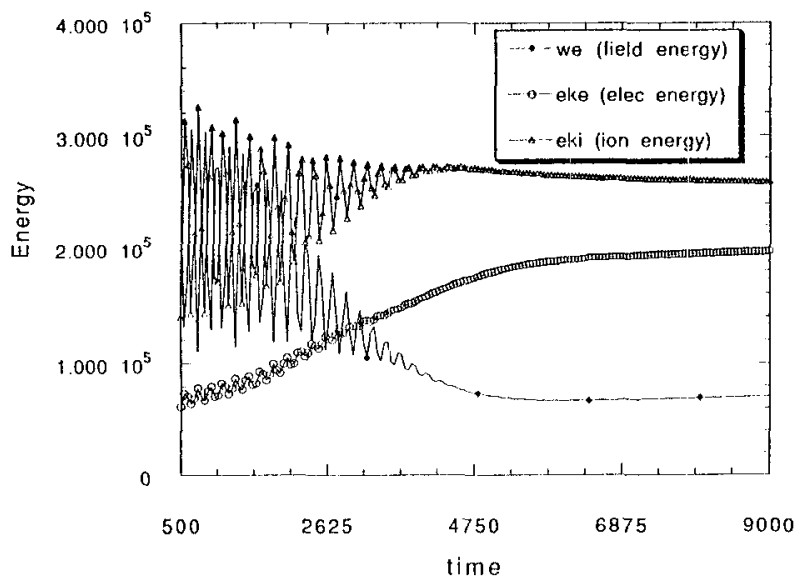

Fig. 2 Time history of simulation energy balance

As the pump wave decays, the energy goes into exciting other modes. Energy is then transferred to the electrons and ions via electron and ion Landau and ion cyclotron damping of the obliquely propagating waves. Substantial heating of both ions and electrons is observed. Figure 2 shows the global energy balance for the same case as shown in Figure 1. The field energy (we) includes all the energy in the electric and magnetic fields of all modes in the simulation; the ion energy (eki) is the total kinetic energy of the ions in the simulations and the electron energy (eke) is the total electron kinetic energy. The large oscillations at early times are due to the exchange of energy between the ions and the fields in the pump Alfvèn wave. Note that at later times, the electrons appear to be responsible for most of the observed damping of the field energy. At late times, the density fluctuations associated with the filamentation decays are observed to damp out. Such electron damping can only be studied with full particle simulation code.

\section{Acknowledgements}

One of the authors (T.K.) was supported by the Max-Planck-Institut für Plasmaphysik (IPP) at Garching and the Max-Planck-Gesellschaft at München, Germany. The work was supported in part by the NSF under Cooperative Agreement No. CCR8809615 and in part by NASA/Heliospheric Physics. A portion of the research described in this paper was carried out at the Jet Propulsion Laboratory, California Institute of Technology, under a contract with the National Aeronautics and Space Administration.

\section{References}

[1] R.D. Ferraro, P.C. Liewer, V.K. Decyk, "A 2D Electrostatic PIC Code for the Mark III Hypercube," Proceedings of 5 th Distributed Memory Computing Conference, Vol. 1, p. 440, IEEE, 1990.

[2] C.K. Birdsall, A.B. Langdon, Plasma Physics via Computer Simulation, McGraw-Hill, New York, 1981.

[3] P.C. Liewer, V.K. Decyk, "A General Concurrent Algorithm for Plasma Particle-in-Cell Simulation Codes," J. Comp. Phys. 85, p. 302, 1989.

[4] P.C. Liewer, E.W. Leaver, V.K. Decyk, J.M. Dawson, "Dynamic Load Balancing in a Concurrent PIC Code on the JPL/Caltech Mark III Hypercube," Proceedings of 5 th Distributed Memory Computing Conference, Vol. 2, p. 939, IEEE, 1990.

[5] A.F. Viñas, M.L. Goldstein, "Parametric Instabilities of Circularly Polarized, Large Amplitude, Dispersive Alfvèn Waves: Excitation of Parallel Propagating Electromagnetic Daughter Waves," to be published in Plasma Physics, 1991.

[6] A.F. Viñas, M.L. Goldstein, "Parametric Instabilities of Circularly Polarized, Large Amplitude, Dispersive Alfvèn Waves: Excitation of Obliquely Propagating Daughter and Sideband Waves," to be published in Plasma Physics, 1991. 